\title{
MicrowaVe HeATING AND JOINING OF CERAMIC CYLINDERS: A MATHEMATICAL MODEL
}

\author{
Michael R. Booty and Gregory A. Kriegsmann \\ Dedicated to Professor Charles G. Lange in fond memory: \\ Teacher, Advisor, Colleague, and Friend.
}

\begin{abstract}
A thin cylindrical ceramic sample is placed in a single mode microwave applicator in such a way that the electric field strength is allowed to vary along its axis. The sample can either be a single rod or two rods butted together. We present a simple mathematical model which describes the microwave heating process. It is built on the assumption that the Biot number of the material is small and that the electric field is known and uniform throughout the cylinder's cross-section. The model takes the form of a nonlinear parabolic equation of reaction-diffusion type, with a spatially varying reaction term that corresponds to the spatial variation of the electromagnetic field strength in the waveguide. The equation is analyzed and a solution is found which develops a hot spot near the center of the cylindrical sample and which then propagates outwards until it stabilizes. The propagation and stabilization phenomenon concentrates the microwave energy in a localized region about the center where elevated temperatures may be desirable.
\end{abstract}

\section{Introduction}

The use of microwaves to sinter or join ceramics is rapidly, gaining acceptance in industry where the efficient production of high quality materials is important. Efficiencies are increased because microwaves penetrate a material and rapidly deposit energy there, in direct contrast to conventional heating schemes where heat diffuses into a material from its surface. However, a disadvantage of microwave heating technology is the need of control systems to prevent thermal runaway and other related instabilities.

The control and dependability of these processes requires a deep understanding of the inherent physics which are described by a formidable nonlinear initial boundaryvalue problem. This system is comprised of the time-harmonic version of Maxwell's equations, the heat equation, an equation of state relating the effective electrical conductivity to the temperature, and a thermal boundary condition on the surface of the ceramic material which balances conduction, convection, and thermal radiation. The

Received June 16, 1994, revised September 14, 1994.

1991 Mathematics Subject Classification: Primary 33C10, secondary 34C10, 34L15.

Key words and phrases: Bessel functions, zeros, monotonicity, inequalities, boundary-value problems, eigenvalues.

The work of the first author was partially supported by the National Aeronautics and Space Administration under NASA Contract No. NAS1-19480 while in residence at ICASE, NASA Langley Research Center, Hampton, VA 23665, and by the National Science Foundation under grant No. DMS9403798.

The work of the second author was supported by the Air Force Office of Scientific Research under grant No. AFOSR 91-0252, the Department of Energy under grant No. DE-FG02-94ER25196, and the National Science Foundation under grant No. DMS-9305828. 
nonlinear character arises from the dependence of the electric field upon the effective electrical conductivity, which is a function of the temperature, the dependence of the temperature upon the microwave power deposition, which is proportional to the product of the effective electrical conductivity and the magnitude of the electric field squared, and the radiative heat loss, which var:es as the fourth power of the temperature. In addition, the boundary-value character of the problem is also challenging because the electromagnetic fields and the ceramic material are confined in a cavity or waveguide applicator of a complicated geometry.

The systematic analysis of these equations under a variety of physical limits has primarily been restricted to one-dimensional geometries (see reference [4] and the bibliography therein), but has recently been extended to three dimensions [4] in the small Biot number limit. However, in all these cases the effect of the waveguide applicator or cavity was neglected, i.e., the ceramic samples were irradiated by plane waves in free space. Nonetheless, the small Biot number theory predicts the phenomenon of thermal runaway and suggests methods for its control.

The problem we model and study in this praper is concerned with the sintering and joining of ceramic fibers in a microwave applicator. As such, it is not described by the theories mentioned above because of the presence of the applicator and also because of the small aspect ratio $a / d$ of the fibers, where $a$ is the radius of the fiber and $d$ is its length. In this paper, we take into account the effects of the applicator by assuming that the electric field is uniform throughout the cylinder's cross-section and known along its length. That is, the ceramic cylinder is thin enough not to perturb the electric field to leading order. Thus, the heating process is modeled by a nonlinear heat equation and boundary condition.

There are three small parameters that arise from a dimensional analysis of the simplified model problem. The first is the aspect ratio defined above, the second is the Biot number $B_{1}$, which is a measure of convective heat loss at the surface, and the third is $B_{2}$, which is a measure of radiative heat loss there. As described above, we have developed an asymptotic theory to study the microwave heating of ceramic slabs and other compact geometries [3,4] as $E_{1} \rightarrow 0$. In these studies $B_{2} \sim B_{1}$ so that the physical effects of both radiation and convection have been incorporated into the theory. This asymptotic theory can be employed to analyze the present problem with the proviso that the parameter $\epsilon^{2}=(a / d)^{2} / B_{1}$ is order one. The net result is that the temperature remains spatially uniform across the ceramic's cross-section and satisfies a nonlinear reaction-diffusion equation along its length. In this equation, the reaction term accounts for adsorption of microwave energy and loss of thermal energy, which arises from convection and radiation at the sample boundaries, and the diffusion coefficient is $\epsilon^{2}$.

Two types of problems naturally arise depending upon the orientation of the ceramic cylinder in the applicator. If the sample is placed so that the electric field has no spatial variation along its axis, then the reaction-diffusion equation has constant coefficients. Equations of this type have received considerable study because of their applicability in a wide variety of physical settings [9]. That the present equation supports traveling transition layers comes as no mathematical surprise. However, in the present physical context it does explain the mechanism for the formation and propagation of hot-spots $[5,6]$ which are seen in experiments $[8,11]$.

On the other hand, if the ceramic fiber is placed so that the electric field varies along its axis, then the equation has a spatially varying reaction term. The analysis and understanding of the solutions to these types of equations are less well understood than 
those described above [1]. We shall analyze such an equation using standard matched asymptotic methods and show how its solution evolves into a hot spot centered on the region of maximum electric field strength. The hot spot then propagates outward from its inception and stabilizes to form a region of elevated temperature. This stable region of elevated temperature can be exploited in fiber sintering and has already been used in joining processes [2]. Moreover, our analysis shows how the size and dynamics of the spot depend upon the temperature dependence of the material's thermal properties. These dependencies are often ignored because the experiments are more sensitive to changes in the effective electrical conductivity with temperature. However, they are essential in understanding the final width of the spot.

\section{Formulation}

A thin cylindrical ceramic sample is positioned in a single mode waveguide applicator, so that the electric field along its axis varies as the fundamental mode of the waveguide. The sample is held in place at its ends by two thermally insulated, microwave transparent push-rods. Although the electric field is altered by the presence of the ceramic, for the present analysis we assume that this effect is negligible and that the time-harmonic electric field is given by

$$
\mathbf{E}=E_{0} \sin (\pi Z / d) \mathbf{j}
$$

where $d$ is also the height of the guide, $E_{0}$ is the strength of the incident mode, and $\mathbf{j}$ is a unit vector perpendicular to the axis of the cylinder. This assumption effectively decouples the equations for the electromagnetic field from the equation for the energy of the sample, and allows us to focus solely on the sample's thermal field. In addition, we assume that the sample is thin enough to ensure that variations in the electric field are negligible across its circular cross-section.

In light of these assumptions, the temperature, $T$, satisfies the energy equation

$$
\frac{\partial}{\partial t}\left(\rho C_{P} T\right)=\nabla \cdot(K \nabla T)+\frac{\sigma(T)}{2} E_{0}^{2} \sin ^{2}(\pi Z / d), \quad 0<Z<d, \quad 0<R<a,
$$

where $R=\sqrt{X^{2}+Y^{2}}$ is the radial distance from the cylinder's axis, $\rho$ is the density of the ceramic, $C_{P}$ is its specific heat, $K$ is its thermal conductivity, and $\sigma$ is its effective electrical conductivity. Although variations of the thermal parameters $K$ and $C_{P}$ are small over the temperature range required for sintering or joining when compared to the change in the electrical conductivity, they are included in the following analysis, since they may have a profound effect on the dynamics of the heating process.

We also require that the temperature satisfies the surface heat balance

$$
K \frac{\partial}{\partial R} T+h\left(T-T_{A}\right)+s e\left(T^{4}-T_{A}^{4}\right)=0, \quad R=a, \quad 0<Z<d,
$$

where $h$ is a constant corresponding to heat loss from the surface by convection, $s$ is a constant measuring radiative heat loss, $e$ is the emissivity of the surface, and $T_{A}$ is the ambient temperature of the surrounding medium. To simplify the analysis that follows, we assume that the ambient temperature remains constant. At the ends of the sample, we prescribe the boundary conditions

$$
\frac{\partial}{\partial Z} T=0, \quad Z=0, d, \quad 0<R<a
$$


and we take the initial temperature of the sample to coincide with the ambient temperature, i.e.,

$$
T(X, Y, Z, 0)=T_{A}
$$

Equations (2)-(4) constitute a nonlinear initial-boundary-value problem for the temperature $T$ within the sample. The nonlinear character of this simplified problem results from the dependence of the electrical conductivity $\sigma$ and the thermal parameters $K$ and $C_{P}$ on the temperature and by the radiative losses at the sample boundary. This is the generalization of the mathematical models for microwave heating as studied by Tian using finite difference simulations [10] and by Kriegsmann using asymptotic methods $[5,6]$.

\section{The simplified theory}

There are three small parameters which arise from a dimensional analysis of equations (2)-(4). The first is the Biot number $B_{1}=h a / K_{A}$, where $K_{A}=K\left(T_{A}\right)$ is the value of the thermal conductivity at the ambient temperature $T_{A}$, and the second is $B_{2}=s e a T_{A}^{3} / K_{A}$. The former is a measure of the relative effects of convection and conduction and the latter is a measure of the relative effects of radiation and conduction. Typical values of $B_{1}$ and $B_{2}$ for ceramics are of the order of 0.01 , see, e.g., [7]. The third small parameter is the aspect ratio of the cylinder $a / d$.

In building our simplified theory, we first intuoduce the dimensionless variables

$$
z=Z / d, \quad u=\frac{T}{T_{A}}-1, \quad \eta=\frac{h}{a\left(\rho C_{P}\right)_{A}} t
$$

where $Z$ is nondimensionalized with respect to the cylinder length , $u$ is the relative deviation of $T$ from $T_{A}$, and $t$ is nondimensicinalized with respect to the ambient convective time $a\left(\rho C_{P}\right)_{A} / h$. Then we define the dimensionless parameters

$$
p=\frac{a \sigma_{A} E_{0}^{2}}{2 h T_{A}}, \quad \alpha=\frac{B_{2}}{B_{1}}, \quad \epsilon^{2}=\frac{(a / d)^{2}}{B_{1}}
$$

and the dimensionless functions

$$
f(u)=\frac{\sigma(T)}{\sigma_{A}}, \quad k=\frac{K(T)}{K_{A}}, \quad \Gamma=\frac{\rho(T) C_{P}(T)}{\left(\rho C_{P}\right)_{A}}
$$

where the subscript $A$ indicates that the quantity is evaluated at $T_{A}$. Here $\alpha$ is the ratio of the convective and radiative Biot numbers and $p$ is a dimensionless power.

Finally, we employ similar methods to those found in References $[3,4]$ to deduce an asymptotic approximation to the solution of (2)-(3) in the limit $B_{1} \rightarrow 0$. The parameters $\alpha, p$, and $\epsilon$ are held fixed in this limiting process. We find that the temperature is given by

$$
T(X, Y, Z, t)=T_{A}\left(1+u(z, t)+O\left(B_{1}\right)\right)
$$


where $u(z, t)$ satisfies the dimensionless initial-boundary-value problem

$$
\begin{aligned}
\frac{\partial}{\partial \eta}(\Gamma(1+u))=\epsilon^{2} \frac{\partial}{\partial z}\left(k \frac{\partial}{\partial z} u\right)+p f(u) \sin ^{2}(\pi z) & \\
-2 & \left\{u+\alpha\left[(u+1)^{4}-1\right]\right\}, \quad 0<z<1 \\
\frac{\partial}{\partial z} u & =0, \quad z=0,1 \\
u(z, 0) & =0, \quad 0<z<1 .
\end{aligned}
$$

We note here that the leading-order approximation to the temperature is independent of the cross-sectional variables but that the $O\left(B_{1}\right)$ correction term depends upon them. The latter gives rise to spatial inhomogeneities which are important in some heating applications. They will not be calculated here.

The nonlinear initial-boundary-value problem (7) constitutes the mathematical statement of our small Biot number theory for the heating of the ceramic rod.

\section{Analysis}

We note here that in some applications where ceramic fibers are sintered in a single mode applicator [11], the parameter $\epsilon$ is very small. For other experiments, such as joining, $\epsilon$ may not be as small. The asymptotic limit $\epsilon \rightarrow 0$ is quite relevant in the former case and is expected to give qualitative results in the later. In mathematical terms, the theory which follows is strictly valid for the ordering $B_{1} \ll \epsilon^{2} \ll 1$. That is, $B_{1} \ll a / d \ll \sqrt{B_{1}}$, so that the diffusion term in (7a) is larger than the neglected terms of $O\left(B_{1}\right)$. Setting $\epsilon=0$ in (7a), we obtain the ordinary differential equation

$$
\frac{\partial}{\partial \eta}(\Gamma(1+u))=p f(u) \sin ^{2}(\pi z)-2\left\{u+\alpha\left[(u+1)^{4}-1\right]\right\},
$$

the solution of which depends upon $z$ parametrically and satisfies the initial conditions (7c).

A reasonable model for the effective electrical conductivity leads to the function $f$ being given by the Arrhenius-like law [12]

$$
f(u)=1+c_{1} e^{-c_{2} / u}
$$

where $c_{1}$ and $c_{2}$ are positive constants. If we fix $z$ and define $P=p \sin ^{2}(\pi z)$, then the solution of (8) and (7c) increases monotonically from its initial value $u=0$ to a terminal value $v$, which is given implicitly by the solution of

$$
P=G(v) \equiv \frac{2\left\{v+\alpha\left[(v+1)^{4}-1\right]\right\}}{f(v)} .
$$

A graph of $G(v)$ is shown in Figure 1, from which we deduce that there can be either one or three solutions of (10) depending upon the value of $P$. If $P<G_{m}$ then the terminal solution $v$ lies on the lower branch, whereas if $P>G_{M}$ then it lies on the upper branch. If $G_{m}<P<G_{M}$, then there are three solutions: one on the upper branch, another on the lower branch, and the third solution on the middle branch. A simple analysis of (8) shows that solutions on the upper and lower branches are stable and that solutions on the middle branch are unstable.

We observe that, because of the spatial variation of the power $P$ along the axis of the waveguide and sample, at different points along its axis, the ceramic sample 


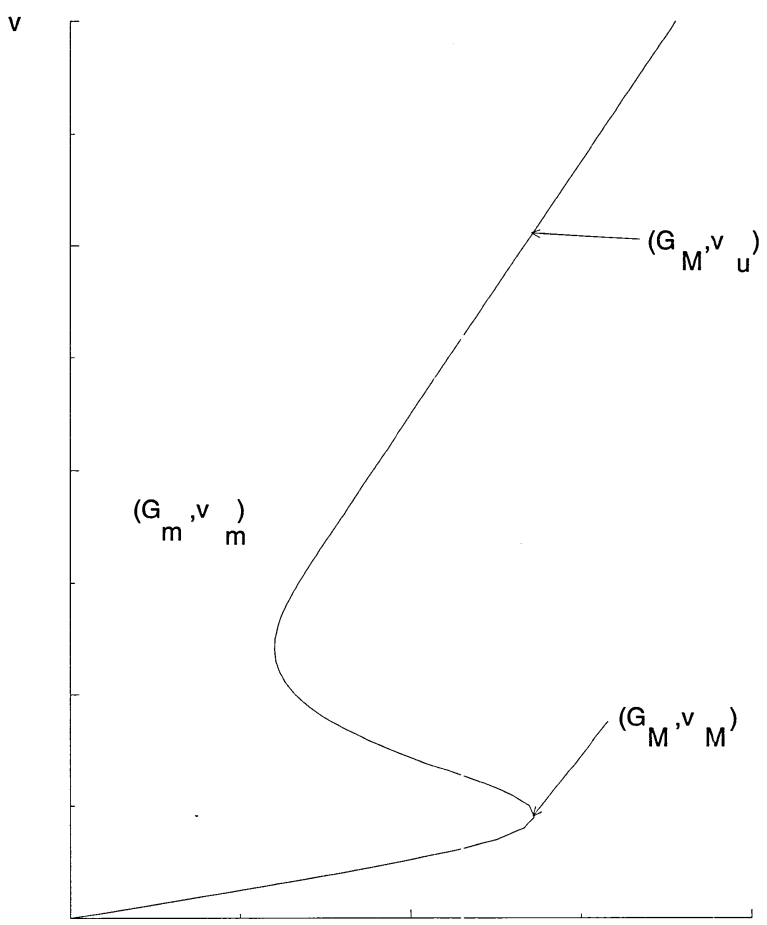

$\mathrm{G}(\mathrm{v})$

Figure 1

experiences different values of $P$, and so there is the possibility that a steady temperature distribution may be on the upper branch in one part of the sample while it is on the lower branch in the remainder. This is indeed the case if we take the maximum dimensionless power $p>G_{M}$. If we define $z_{1}$ and $z_{2}$ by

$$
z_{1}=\frac{1}{\pi} \arcsin \left(\sqrt{G_{M} / p}\right), \quad z_{2}=1-z_{1},
$$

then $P>G_{M}$ in the interval $z_{1}<z<z_{2}$, and a steady temperature distribution in that part of the sample must lie on the upper branch. We have sketched this in Figure 2 .

If we try to resolve the discontinuity in this steady state approximation by introducing boundary layers at $z_{1}$ and $z_{2}$, within which the diffusion term of (7a) is important, then we are immediately struck with the discoricerting fact that such a solution does not exist. To see this, we introduce the stretched variable $\bar{z}=\left(z-z_{1}\right) / \epsilon$ into (7a), set $\frac{\partial}{\partial \eta}=0$, and obtain

$$
\frac{d}{d \bar{z}}\left(k \frac{d}{d \bar{z}} u\right)+f(u)\left[G_{M}-G(u)\right]=0, \quad|\bar{z}|<\infty,
$$

where $G$ is defined in (10). The boundary conditions for (12) are clearly $u \rightarrow v_{u}$ as $\bar{z} \rightarrow \infty$, where $v_{u}$ is the value of $u$ on the upper branch corresponding to $G_{M}$ (see Figure 1), and $u \rightarrow v_{M}$ as $\bar{z} \rightarrow-\infty$. Thus, $u^{\prime} \rightarrow 0$ as $|\bar{z}| \rightarrow \infty$. Upon multiplying 


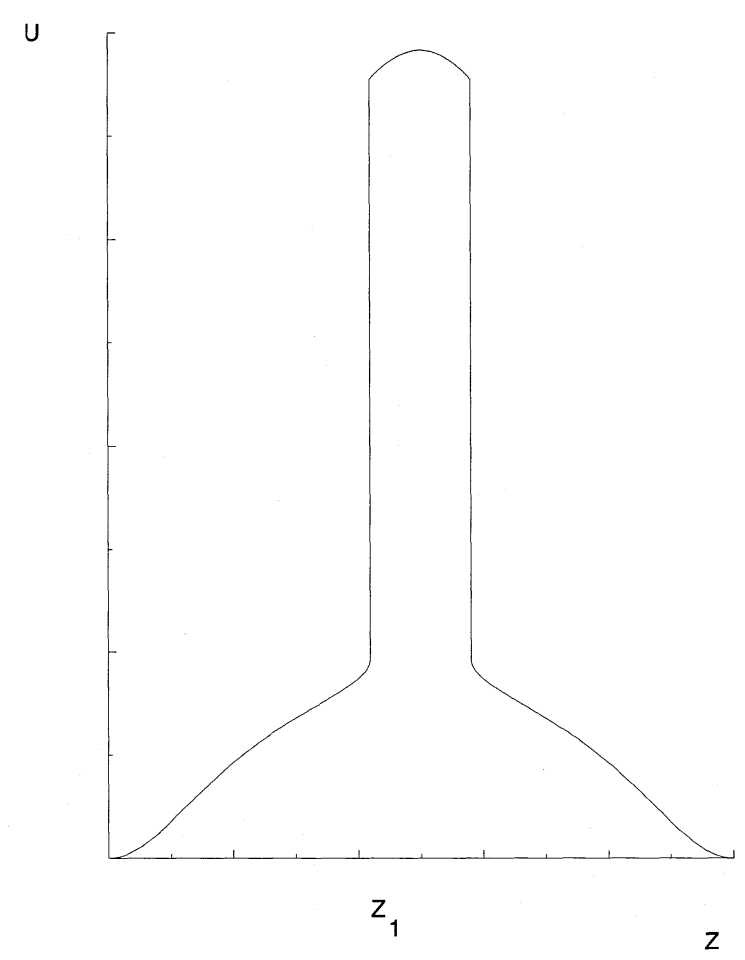

Figure 2

(12a) by $k \frac{d}{d \bar{z}} u$, integrating the result from $\pm \infty$, and using $u^{\prime} \rightarrow 0$ as $|\bar{z}| \rightarrow \infty$, we obtain

$$
\int_{v_{M}}^{v_{u}} k(u) f(u)\left[G_{M}-G(u)\right] d u=0
$$

However, from the definition of $G_{M}$, we deduce that the integrand is positive so that no heteroclinic connection exists.

We resolve this apparent contradiction by removing the constraint that $\frac{\partial}{\partial \eta}=0$ in the above analysis. That is, we shall look for a traveling wave solution of $(7 \mathrm{a})$ which has the form

$$
u=\phi(\bar{z}), \quad \text { where } \quad \bar{z}=\frac{z-z_{1}-C(\tau)}{\epsilon} \quad \text { and } \quad \tau=\epsilon \eta .
$$

Inserting this ansatz into (7a), we obtain, at leading order,

$$
\frac{d}{d \bar{z}}\left(k \frac{d}{d \bar{z}} \phi\right)+C^{\prime} \frac{d}{d \bar{z}}(\Gamma(1+\phi))+f(\phi)\left[p \sin ^{2} \pi\left(z_{1}+C\right)-G(\phi)\right]=0
$$

where the prime on $C$ denotes a derivative with respect to its slow time argument $\tau=\epsilon \eta$, and the equation is to be solved on the interval $-\infty<\bar{z}<\infty$. We deduce similar boundary conditions to the above and find again that, as in (12b),

$$
\frac{d}{d \bar{z}} \phi \rightarrow 0 \quad \text { as } \quad \bar{z} \rightarrow \pm \infty
$$


Now, however, asymptotic matching implies that the solution $\phi$ has the limits

$$
\phi \rightarrow \phi_{ \pm}(C) \text { as } \quad \bar{z} \rightarrow \pm \infty
$$

where $\phi_{+}(C)>\phi_{-}(C)$ are those roots of $p \sin ^{2} \pi\left(z_{1}+C\right)=G(\phi)$ which lie on the upper and lower branches of the S-shaped response curve of (10), respectively. Finally, we observe that the boundary-value problem (15a)-(15c) is not well-posed because of its translational invariance in $\bar{z}$. To remove this ambiguity, we choose

$$
\phi(0)=\frac{1}{2}\left[\phi_{+}(C)+\phi_{-}(C)\right] .
$$

Multiplying (15a) by $k \frac{d}{d \bar{z}} \phi$, integrating the resulting expression with respect to $\bar{z}$, and applying (15b) and $(15 \mathrm{c})$, we deduce that

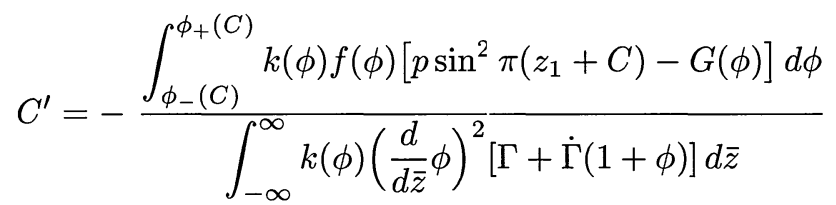

where the dot above $\Gamma$ denotes its derivative with respect to $\phi$.

This equation is a first-order nonlinear ordinary differential equation for the position of the slowly moving traveling wave, or front, which has the initial condition $C(0)=0$ and connects the solutions on the upper and lower branches of (10). We note that the dependence of the right-hand-side on $C$ is implicit and, in particular, the integrand in the denominator depends upon $C$ through the solution of the boundary-value problem (15).

We now can deduce the dynamics of the heating process qualitatively by considering (16) when $p>G_{M}$. Initially, the rod heats up according to (8), so that a discontinuous temperature profile begins to form with discontinuities at $z=z_{1}$ and $z=z_{2}$ and a hot spot on the interval $z_{1}<z<z_{2}$, as shown in Figure 2. At this time, considering the dynamics of the left half of the sample, $0<z<1 / 2$ alone, since the solution is symmetric about the sample's midpoint, $C=$ J, and, from (16), $C^{\prime}$ is negative if we assume that the term $\Gamma+\dot{\Gamma}(1+\phi)$ is positive, as is the case for physically realistic applications. Thus, $C$ decreases and the front begins to move to the left, from $z=z_{1}$, on the slow time scale $\tau=\epsilon \eta$. This elevates a larger portion of the rod to the higher temperatures of the upper branch, i.e., the hot spot begins to grow.

This description is given under the proviso that

$$
\frac{d}{d \phi} \Gamma(\phi)(1+\phi)>0
$$

which, from the definitions of $\phi$ and $\Gamma$, is equivalent to the statement that the internal energy density of the ceramic, $\rho C_{P} T$, is an ircreasing function of the temperature. This is true in ceramics and in almost all materials, away from phase transitions.

We now turn to the further development and stabilization of the hot spot. Noting the definitions of $\phi_{ \pm}(C)$, it follows that the derivative with respect to $C$ of the numerator on the right hand side of (16), i.e.,

$$
N=\int_{\phi_{-}(C)}^{\phi_{+}(C)} k(\phi) f(\phi)\left[p \sin ^{2} \tau^{2}\left(z_{1}+C\right)-G(\phi)\right] d \phi,
$$




$$
\frac{\partial}{\partial C} N=\pi p \int_{\phi_{-}(C)}^{\phi_{+}(C)} k(\phi) f(\phi) \sin 2 \pi\left(z_{1}+C\right) d \phi
$$

which is strictly positive, since $0<z_{1}+C<1 / 2$, and $k$ and $f$ are positive. Also, since $\phi_{+}(0)=v_{u}, \phi_{-}(0)=v_{M}$, and $G_{M}=p \sin ^{2}\left(\pi z_{1}\right)$, we deduce from (17) that $N(0)>0$. Similarly, if we define $\tilde{C}$ so that $G_{m}=p \sin ^{2} \pi\left(z_{1}+\tilde{C}\right)$, then $\phi_{+}(\tilde{C})=v_{m}$, $\phi_{-}(\tilde{C})$ is the corresponding point on the lower branch of the S-shaped curve, and, consequently, $N(\tilde{C})<0$. Hence by continuity of $N$ with respect to $C$, there is a unique $C=C_{*}<0$ such that $N\left(C_{*}\right)=0$. Since the denominator $D(C)$ of (16) is, for the reasons given above, strictly positive, we deduce that the solution of the differential equation $C^{\prime}=-N(C) / D(C)$ with initial condition $C(0)=0$ is monotone decreasing and tends to $C_{*}$ as $\tau \rightarrow \infty$, with its final approach being exponential in $\tau$. Thus, the hot spot grows in size and finally stabilizes to occupy the region $z_{1}+C_{*}<z<1-z_{1}-C_{*}$.

We now can consider the influence of a temperature-dependent thermal conductivity on the equilibrated value $C_{*}$. First, we recall that $C_{*}$ is such that

$$
N\left(C_{*}\right)=\int_{\phi_{-}\left(C_{*}\right)}^{\phi_{+}\left(C_{*}\right)} k(\phi) f(\phi)\left[p \sin ^{2} \pi\left(z_{1}+C_{*}\right)-G(\phi)\right] d \phi=0,
$$

and consider the case when the thermal conductivity is constant, so that $k(\phi)=1$. Then, since $\phi_{-}(C)$ and $\phi_{+}(C)$ are such that the local power at the boundaries of the hot spot is $P=p \sin ^{2} \pi\left(z_{1}+C_{*}\right)=G\left(\phi_{ \pm}\left(C_{*}\right)\right)$, the graphs of $f(u) G(u)$ and $\operatorname{Pf}(u)$ intersect transversally at $u=\phi_{-}\left(C_{*}\right), u=\phi_{+}\left(C_{*}\right)$, and, from (19), also at some value between, i.e., on the interval $\phi_{-}\left(C_{*}\right)<u<\phi_{+}\left(C_{*}\right)$. Equation (19) implies that the unique value $C_{*}$ is such that the areas of the two lobes between the graphs of $f(u) G(u)$ and $P f(u)$ are equal. When $k(\phi)$ is not constant but, for example, is a monotone increasing function of $\phi$ with $k(0)=1$, as is the case for typical ceramics, the influence of the temperature-dependence with $C$ fixed is such as to increase the area of the right-hand lobe (at larger $\phi$ ) more than that of the left-hand lobe. This increases $N(C)$, so that, since $\frac{\partial}{\partial C} N>0$, the temperature-dependence of $k$ is such as to decrease the equilibrating value $C_{*}$ to more negative values, and hence increase the final width of the hot spot in the steady state.

\section{Numerical examples}

To illustrate the analysis presented in the previous section quantitatively, we use numerical means to solve the two-point boundary-value problem (15) that determines $C(\tau)$. Although (15) could be solved by a straightforward shooting method, some care must be exercised to avoid the exponential growth in $\bar{z}$ of solutions to (15a) as the saddle points of the heteroclinic connection are approached. The procedure we developed to solve (15) has two parts: first, we restate the problem in the form of two coupled initial-value problems on the interval $\bar{z}>0$, and then we use a numerical shooting method to determine $C^{\prime}$ as a function of $C$. Once this is achieved, $C(\tau)$ can be determined by numerical quadrature. 
The coupled pair of initial-value problems replacing $(15 \mathrm{a}, \mathrm{d})$ are

$$
\begin{aligned}
\frac{d}{d \bar{z}}\left(k \frac{d}{d \bar{z}} \psi_{1}\right)+C^{\prime} \frac{d}{d \bar{z}}\left(\Gamma\left(1+\psi_{1}\right)\right) & +f\left(\psi_{1}\right)\left[p \sin ^{2} \pi\left(z_{1}+C\right)-G\left(\psi_{1}\right)\right]=0 \\
\frac{d}{d \bar{z}}\left(k \frac{d}{d \bar{z}} \psi_{2}\right)-C^{\prime} \frac{d}{d \bar{z}}\left(\Gamma\left(1+\psi_{2}\right)\right) & +f\left(\psi_{2}\right)\left[p \sin ^{2} \pi\left(z_{1}+C\right)-G\left(\psi_{2}\right)\right]=0 \\
\psi_{1}(0)=\psi_{2}(0) & =\frac{1}{2}\left[\phi_{+}(C)+\phi_{-}(C)\right] \\
\psi_{1}^{\prime}(0) & =-\psi_{2}^{\prime}(0)=\gamma
\end{aligned}
$$

where (20b) arises from reflection of the original equation (15a) and the interval $\bar{z}<0$ about $\bar{z}=0$, and the parameter $\gamma$ is to be detiermined. These equations are solved for fixed $C$ with $\tilde{C}<C<0$ on the finite interval $0<\bar{z}<L$ where $L$ is taken to be sufficiently large. The function $C^{\prime}$ and the parameter $\gamma$ depend on $C$, and are determined by demanding that

$$
\begin{array}{ll}
\psi_{1}^{\prime}-\lambda_{1}\left(\psi_{1}-\phi_{+}(C)\right):=0, & \bar{z}=L, \\
\psi_{2}^{\prime}-\lambda_{2}\left(\psi_{2}-\phi_{-}(C)\right):=0, & \bar{z}=L,
\end{array}
$$

where $\lambda_{1}$ and $\lambda_{2}$ are the negative eigenvalues corresponding to exponential decay toward the saddle points as $\bar{z} \rightarrow \infty$. Once $\psi_{1}$ and $\psi_{2}$ are determined, the solution to (15) is given by $\psi_{1}(\bar{z})$ when $\bar{z}>0$ and by $\psi_{2}(-\bar{z})$ when $\bar{z}<0$.

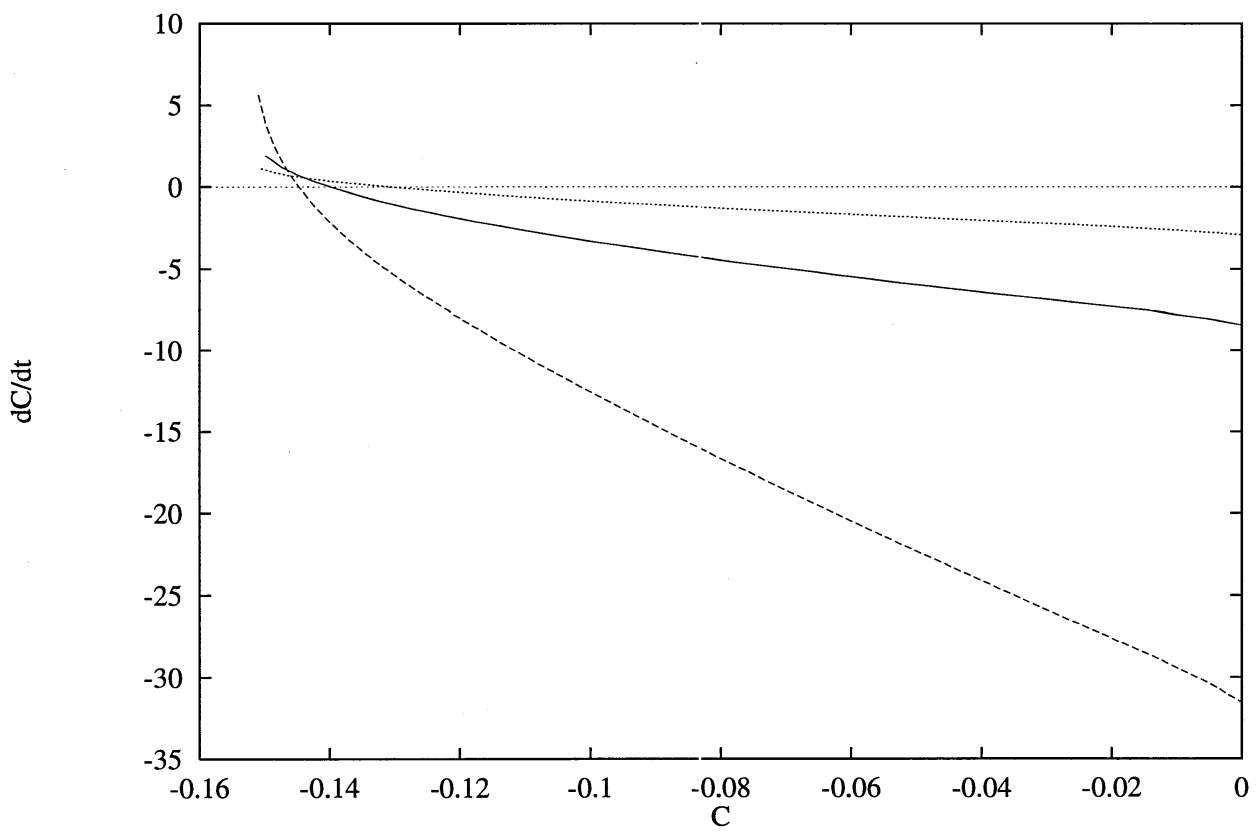

FiguRE 3

The numerical implementation of (20)-(21) :s straightforward and will not be discussed here. We have set $\Gamma=1$ in the calculations in order to isolate the effects of the temperature dependent thermal conductivity, $k(\phi)$. Furthermore, we have fixed the values of $c_{1}$ and $c_{2}$ in (9) to be 500 and 13.7, respectively [12], and we have taken the values of the parameters $p$ and $\alpha$ of (5b) to be $p=9$ and $\alpha=0.05$. The interval 
for $C$ is given by $\tilde{C}=(1 / \pi) \arcsin \left(\sqrt{G_{m} / p}\right)-(1 / \pi) \arcsin \left(\sqrt{G_{M} / p}\right)<C<0$, which insures the existence of $\phi_{ \pm}$.

In Figure 3, the solid curve shows the functional dependence of $C^{\prime}$ on $C$ for the case $k=1$, and the dashed curve corresponds to

$$
k(\phi)=1+\frac{1}{2} \phi^{2}
$$

which models a material whose thermal conductivity increases with temperature. The value of $C_{*}$ for this case is less than that for $k=1$, and this is in agreement with our discussion at the end of the previous section. Finally, the dotted curve in Figure 3 corresponds to

$$
k(\phi)=e^{-\phi / 2},
$$

which models a material whose thermal conductivity decreases with temperature. The value of $C_{*}$ for this case is greater than that for $k=1$.

The dynamical evolution of $C(\tau)$ readily follows from the functional dependence of $C^{\prime}$ on $C$. The results, showing $|C|$ plotted against $\tau$, are given in Figure 4 where the solid, dashed, and dotted curves correspond to the three thermal conductivities just discussed. In each case, $C(\tau)$ decreases monotonically from 0 to its respective value of $C_{*}$.

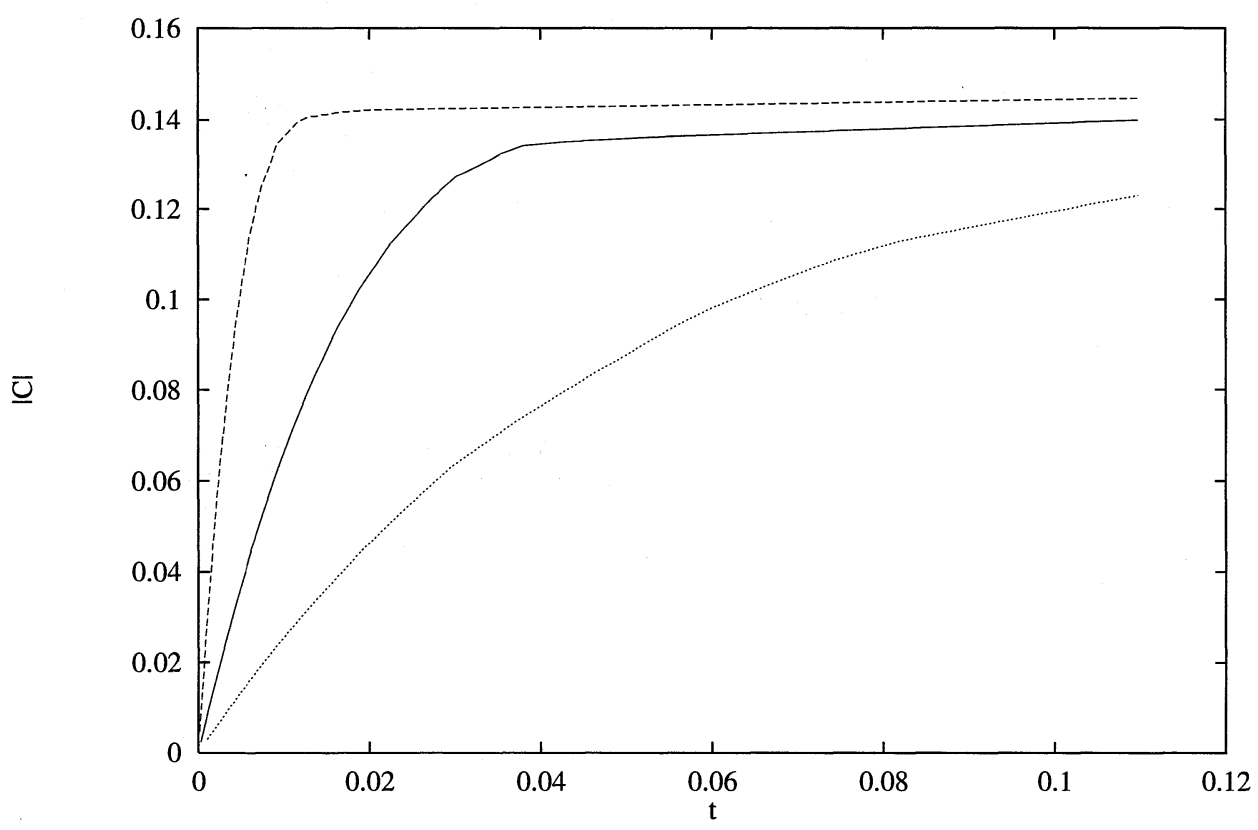

FIGURE 4

\section{Conclusion}

The implications of the analysis for the sintering of ceramic fibers and joining of ceramic cylinders is now evident. In the first case, the hot spot forms, propagates, and then stabilizes. If the temperature in the relatively warm region of the hot spot is sufficient for sintering, then the fiber can be slowly pulled through the guide, thus 
insuring that the entire sample is processed. This is used as a means of sintering ceramics in practice [11], and the rate at which the fiber is to be drawn is found experimentally. In the second case, the hot spot is to encompass the butt joint at which the two ceramic cylinders are to be joined, and, if the temperature in this region is sufficient for the materials to fuse, then a strong joint can be obtained [2].

We close by briefly describing another type of solution that is possible if the applied electric field has a minimum at the center of the fiber. This may occur by exciting the applicator in one of its higher spatial modes. If the maximum of the electric field is such that $P>G_{M}$ and the minimum is such that $P<G_{M}$, then hot spots will form at both ends of the fiber. These spots will grow in size and stabilize according to the mechanism described at the end of the last section.

\section{References}

1. P. C. Fife, Dynamics of Internal Layers and Diffusive Interfaces, CBMS-NSF Regional Conference Series vol. 35, SIAM, Philadelphia, 1988.

2. H. Fukushima, T. Yamanaka, and M. Matsui, Microwave heating of ceramics and its applications to joining, Material Research Society Symposium Proceedings, Vol. 124, 1988.

3. G. A. Kriegsmann, Thermal runaway in microwave heated ceramics: a one-dimensional model, J. Appl. Phys., 71 (1992), 1960-1966.

4. _ Microwave heating of dispersive media, SlAM J. Appl. Math., 53 (1993), 655-669.

5. - Formation of hot spots in microwave healed ceramic rods, In: Microwaves: Theory and Applications in Material Processing II, Ceramic .Transactions Vol. 36, The American Ceramic Society, Westerville, Ohio, 1993.

6. _ـ Growth and stabilization of hot spots in nicrowave heated ceramic fibers, Material Research Society Symposium Proceedings, Vol. 270, San Francisco, 1994, in press.

7. F. P. Incropera and D. P. DeWitt, Introduction to Heat Transfer, Wiley, New York, 1985.

8. D. L. Johnson, Hot spots in microwave heated fivers, growth and stabilization of hot spots in microwave heated ceramic fibers, Material Research Society Symposium Proceedings, Vol. 270, San Francisco, 1994, in press, MRS 94.

9. J. J. Tyson and J. P. Keener, Singular perturbation theory of traveling waves in excitable media (a review), Phys. D, 32 (1988), 327-361.

10. Y. L. Tian, Practices of ultra-rapid sintering of ceramics using single mode applicators, In: Microwaves: Theory and Applications in Microwave: Processing, Ceramic Transactions, 21 (1991), 283-300.

11. G. J. Vogt and W. P Unruh, Microwave hybrid heat:ing of alumina filaments, In: Microwaves: Theory and Applications in Material Processing II, Ceramic Transactions Vol. 36, The American Ceramic Society, Westerville, Ohio, 1993.

12. D. G. Watters, An Advanced Study of Microwave Sintering, Ph.D. Thesis, Northwestern University, Evanston, Il., 1988.

Department of Mathematics, Center for Applied Mathematics and Statistics, New Jersey Institute of Technology, University Heights, Newark, NJ 07102, U.S.A. 